\title{
PYODERMA GANGRENOSUSM ARISING IN ASSOCIATION WITH CROHN'S COLITIS AND DIABETES MELLITUS
}

\author{
Broshtilova V., S. Marina \\ Department of Dermatology and Venereology, Faculty of Medicine, Sofia Medical University
}

Reviewed by: Prof. Kr. Ivanov

\begin{abstract}
A 36-year-old man presented with a large ulceration with elevated cribriform borders on his right lower leg dated one month ago. The patient's past history showed a four-year history of bowel inflammatory disease in remission since two years, and badly controlled diabetes mellitus with persistently elevated blood glucose despite insulin supplementation since one year. The clinical and histology findings were consistent with pyoderma gangrenosum. Pathogenic therapy was introduced to achieve full control of his skin symptoms. Pyoderma gangrenosum is a common skin complication and manifestation of Crohn's disease. It could rarely occur in patients with insulin-dependent diabetes. Remarkably, the constellation of inflammatory bowel disease, diabetes and pyoderma gangrenosum is anecdotal and may suggest susceptibility to multiple autoimmune syndrome. Herein, this unique case of complicated autoimmune disturbance is reported to point out the importance of Th1 stimulated regulatory mechanism in formation of skin, intestinal and glandular disorders.
\end{abstract}

\section{CASE REPORT}

A 36-year-old man presented with a painful, ulcero-necrotic lesion on his right lower leg which appeared 8 weeks ago. On an outpatient basis the large erythematous ulcer with draining pustules was interpreted as a pyogenic inflammatory process, although a culture yielded rare colonies of coagulase-negative Staphylococcus aureus. Cephalexin and amoxicillin-clavulanic acid were ineffective, and broad surgical excision was undertaken. The lesion rapidly worsened with the appearance of erythematous patches overlied by putules on the site of surgery. The patient's general status deteriorated and he was transferred to the Department of Dermatology for diagnostic verification and appropriate treatment.

The patient had a two-year history of insulin-dependent diabetes mellitus, which was managed with intensified combined regimen; at the most recent evaluation the glycosylated hemoglobin value was elevated, at 9.9 percent. Four years ago, the patient had to pass four or five soft stools daily, and by colonoscopy he was diagnosed to have Crohn's disease. He was treated with sulfasalazine and obtained full remission which was sustained to date. The patient had iron-deficiency anemia, which was ascribed to the latent chronic inflammatory bowel disease. He had no family history, and refused to take any medications.

On physical examination, the temperature was $37.6^{\circ} \mathrm{C}$, and the blood pressure was $110 / 75 \mathrm{~mm} \mathrm{Hg}$. On the right leg there was an indurated large plaque with central ulceration and elevated undermined border with draining pustules on it (Fig.1). The diameter of the lesion was 10 by $15 \mathrm{~cm}$. On former excision lines multiple tiny pustules confluent to large infiltrated plaques were seen (Fig.2). The peripheral pulses were intact.

The results of hematologic and blood chemical tests were in normal ranges, except for low hemoglobin level (101 g/l) and elevated erythrocyte sedimentation rate $(99 \mathrm{~mm} / \mathrm{h})$. A culture of the wound yielded a few colonies of coagulase-negative staphylococci; three blood cultures were sterile. A fungal wet preparation, and fungal and mycobacterial cultures of the lesion on the leg were negative. Radiographs of the right ankle showed no evidence of osseous involvement.

Examination of a superficial punch-biopsy specimen from the lesion on the right foot showed pseudoepitheliomatous hyperplasia with intraepidermal neutrophilic abscesses and spongiosis of the adjacent epidermis. In the dermis a mixed diffuse superficial and deep inflammatory infiltrate containing numerous neutrophils was present. There was no evidence of vasculitis in the specimen. The absence of massive subepidermal edema and the presence of ulceration ruled out Sweet's syndrome. In conjunction with the appropriate clinical findings, the histologic findings were consistent with the diagnosis of pyoderma gangrenosum.

The patient was treated with 6-methylprednisolon in combination with sulfasalazine $2 \mathrm{~g}$ daily as a corticosteroidsparing agent. Wet dressings were applied on the ulcer. On the follow-up visit at the 6 week of therapy the patient still had a small ulcer measured $4 \mathrm{~cm}$ in diameter (Fig.3). An atrophic, hypopigmented scar marked the former inflammatory process. 


\section{DISCUSSION}

Skin lesions that mimic a pyogenic infection but result from a sterile inflammatory process involving neutrophils form the spectrum of the neutrophilic dermatoses (1). Usually the clinical characteristics of the lesions fully imitate a pyogenic process. The progressive course of the disease and the treatment refractivity to antibacterial therapy, together with the negative results of microbial cultures, verify the rightful diagnosis.

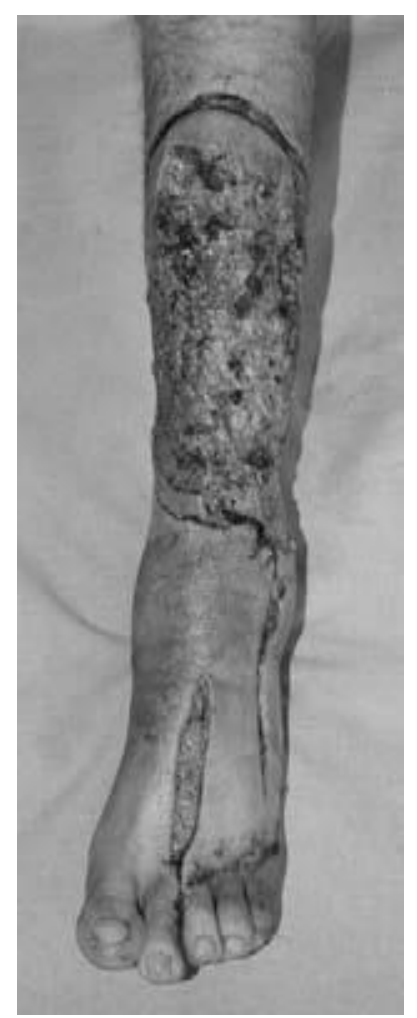

Fig. 1. An indurated large plaque with central ulceration and elevated undermined border with draining pustules on it.

The two most common forms of neutrophilic dermatosis are pyoderma gangrenosum and Sweet's syndrome (2), each of which may be idiopathic or related to an underlying systemic disease. Pyoderma gangrenosum most often appears as pustules on the lower legs. Ulcers with undermined violaceous borders evolve from the initial erythemo- infiltrative plaques. Pyoderma gangrenosum is often associated with inflammatory bowel disease (1 in 3 cases). Sweet's syndrome usually affects arms and face with nonpustular, solid papules or plaques. It rarely ulcerates and is not so often associated with Crohn's disease (1 in 10 cases). A syndrome with overlapping features of these two disorders has been designated atypical pyoderma gangrenosum, which is characterized by superficial pustules or vesicles and bullae that tend to heal readily without scarring (3). Our patient showed classic features of pyoderma gangrenosum.

There are four types of pyoderma gangrenosum: ulcerative, pustular, bullous, and vegetative (4). The ulcerative variety is the most common. The lesions often begin as small, painful pustules with an erythematous halo. Over a period of a few days, some of them enlarge rapidly and ulcerate, with a well-defined, violaceous, undermined border and often an intensely erythematous halo about $2 \mathrm{~cm}$ in diameter. These lesions are extremely painful. Pustular pyoderma gangrenosum is often associated with inflammatory bowel disease. The pustules are painful, and often surrounded by erythema. This type of pyoderma gangrenosum has also been seen after jejunoileal bypass surgery for morbid obesity. The bullous type of pyoderma gangrenosum is very often associated with myeloproliferative diseases such as myelogenous leukemia, dysmyelopoietic anemia, and polycythemia rubra vera (5).

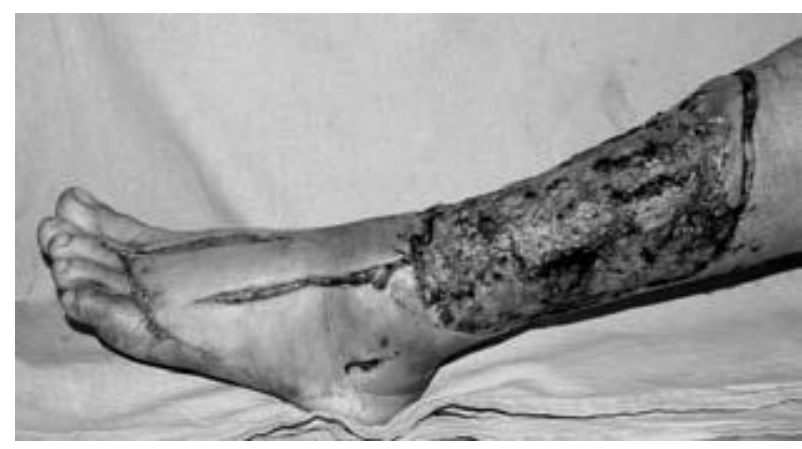

Fig. 2. Excision lines multiple tiny pustules confluent to large infiltrated plaques were seen

Vegetative pyoderma gangrenosum appears as a superficial ulcer without an undermined border, progresses slowly, is not very painful, and is usually solitary. It is usually not associated with other diseases and is generally very responsive to therapy $(5,6)$.

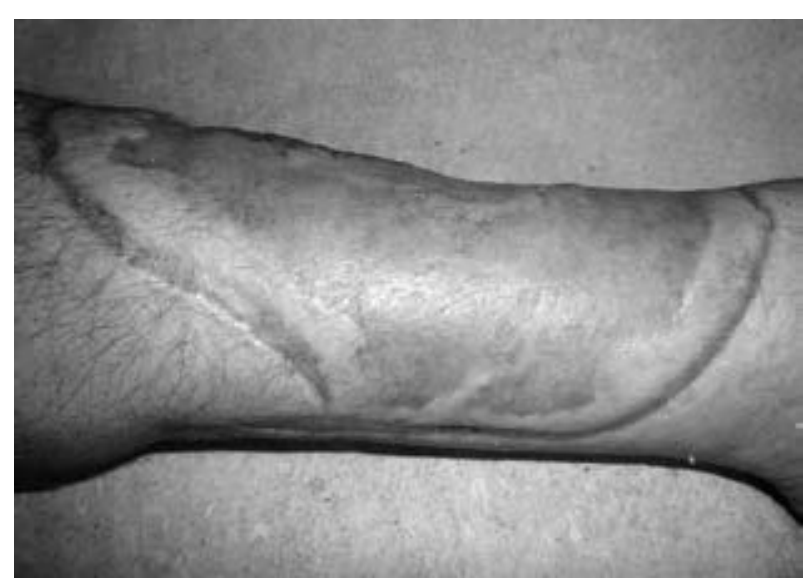

Fig. 3. A 2-month follow-up

Although many abnormalities of neutrophil function have been reported, the pathogenesis of pyoderma gangrenosum remains elusive. It can occur in patients who are receiving immunosuppressive therapy for organ transplantation or chemotherapy for leukemia, as well as in patients who are infected with the human immunodeficiency virus. Although neutrophils extensively infiltrate the central portion of the lesions, the erythematous halo has a lymphocytic infiltrate (7). In pyoderma gangrenosum cases, impaired 
T-cell regulation of cutaneous inflammation may be the culprit, since skin-homing $\mathrm{T}$ cells have a key role in cutaneous inflammation and immune surveillance.

An important feature of either type of neutrophilic dermatosis is pathergy, the appearance of the disorder in an area of normal skin that has been subjected to trauma (in this patient, the surgical incision). If pyoderma gangrenosum is misinterpreted as an infection that requires surgical debridement, larger areas of the disease can develop as a pathergic reaction each time the procedure is performed. Even taking skin from elsewhere on the body for grafting at the site of an ulcer can result in the appearance of a lesion at the donor site.

The disorders that are most commonly associated with pyoderma gangrenosum are inflammatory bowel diseases, with ulcerative colitis and Crohn's disease occurring at equal frequencies. The appearance of the skin lesions may precede the clinical onset of the bowel disease, or may appear years after the onset of the systemic process. Seronegative polyarthritis and rheumatoid arthritis are often associated with pyoderma gangrenosum. Pyoderma gangrenosum is occasionally associated with collagen vascular diseases, including Takayasu's arteritis, systemic lupus erythematosus, and Behcet's disease, which in itself is a disorder involving neutrophilic inflammation of the skin and other tissues, such as mucous membranes, nerve tissue, and tissues of the eye and brain. Various other disorders are occasionally associated with pyoderma gangrenosum $(2,4,6)$.

Diabetes increases the risk of infection and blisters, usually on the legs (bullosis diabeticorum) but, to date there is no convincing data that it increases the risk of pyoderma gangrenosum. Blister formation is probably a sequela of trauma in diabetic patients who have microvascular disease and whose diabetes is severe, requiring insulin therapy. The blisters of bullosis diabeticorum are typically noninflammatory, without surrounding erythema. An unruptured bulla can evolve into a pustule, but diabetes would not explain this patient's lesions. Certainly the vascular involvement in diabetes could deteriorate and cause progression of the ulceration in pyoderma gangrenosum. Surprisingly, we found no data of prominent endothelial thickening of the small vessels in the dermis, and evidence of vasculopathy in our patient's skin biopsy.

Systemic corticosteroids are currently the standard of therapy, but long-term use can have adverse effects. Nonsteroidal drugs that may be administered include dapsone and sulfasalazine, which are generally less effective when used alone than when used as agents that allow a reduction of the dose of corticosteroids. Immunosuppressive agents such as tacrolimus, mercaptopurine, azathioprine, mycophenolate mofetil, methotrexate, chlorambucil, cyclophosphamide, and especially cyclosporine have been beneficial in some cases. Cofazimine is sometimes very effective. Minocycline and colchicine, both of which probably affect neutrophils by acting as antiinflammatory agents, are sometimes helpful. Plasmapheresis and intravenously administered immune globulin have also been reported to be effective. Thalido- mide has helped some patients; this drug increases the proliferation and migration of keratinocytes, which may aid in the healing of large ulcers $(8,9)$. Surprisingly, large ulcerated areas of pyoderma gangrenosum usually heal without skin grafting. If skin grafting is necessary in a patient with pyoderma gangrenosum, it should not be performed until active disease has been suppressed by appropriate therapy. Secondary infection, if present, should be treated. Paradoxically, sometimes the antibiotic therapy could have beneficial effect due to the fact that antibiotics often inhibit the chemotaxis of neutrophils, resulting in a nonsteroidal antiinflammatory effect (10).

Finally, in cases of pyoderma gangrenosum associated with inflammatory bowel disease, effective treatment of the bowel disease often results in resolution of the pyoderma gangrenosum, especially if it is the pustular variety. In some cases, however, surgical resection of all the involved segments of the bowel has failed to result in resolution of the pyoderma gangrenosum.

\section{REFERENCES}

1. Wolff K, Sting1 G. Pyoderma gangrenosum. In: Freedberg IM, Eisen AZ, Wolff K, et al., eds. Fitzpatrick's dermatology in general medicine. $5^{\text {th }}$ ed. Vol. 1. New York: McGraw-Hill, 1999:1140-8.

2. Lear JT, Atherton MT, Byrne JP. Neutrophilic dermatoses: pyoderma gangrenosum and Sweet's syndrome. Postgrad Med J 1997;73:65-68.

3. Bennett ML, Jackson JM, Jorizzo JL, Fleischer AB, White WL, Callen JP. Pyoderma gangrenosum: a comparison of typical and atypical forms with an emphasis on time to remission: case review of 86 patients from 2 institutions. Medicine (Baltimore) 2000;79:37-46.

4. Powell FC, Su WPD, Perry HO. Pyoderma gangrenosum: classification and management. $\mathrm{J} \mathrm{Am}$ Acad Dermatol 1996;34:395-409.

5. Caughman W, Stern R, Haynes H. Neutrophilic dermatosis of myeloproliferative disorders: atypical forms of pyoderma gangrenosum and Sweet's syndrome associated with myeloproliferative disorders. $J$ Am Acad Dermatol 1983;9:751-758.

6. Su WPD, Schroeter AL, Perry HO, Powell FC. Histopathologic and immunopathologic study of pyoderma gangrenosum. J Cutan Pathol 1986;13:323-330.

7. Robert C, Kupper TS. Inflammatory skin diseases, T cells, and immune surveillance. $N$ Engl J Med 1999;341:1817-1828.

8. Federman GL, Federman DG. Recalcitrant pyoderma gangrenosum treated with thalidomide. Mayo Clin Proc 2000;75:842-844.

9. Nasca MR, O'Toole EA, Palicharla P, West DP, Woodley DT. Thalidomide increases human keratinocyte migration and proliferation. $J$ Invest Dermatol 1999;113:720-724

10. Casellas F, Borruel N, Papo M, et al. Antiinflammatory effects of enterically coated amoxicillin-clavulanic acid in active ulcerative colitis. Inflamm Bowel Dis 1998;4:1-5. 